therapy at admission. In addition, mechanical ventilation and multiple organ failure were risk factors for mortality at 1 year.

Disclosure of Interest: None declared

DOI: 10.1136/annrheumdis-2017-eular.3692

\section{SAT0570 AN IGNORED DISEASE IN ADULTS: ACUTE RHEUMATIC FEVER}

B. Bitik $^{1}$, O. Kucuksahin ${ }^{2}$, N. Karahan Yesil ${ }^{1}$, H. Yesil ${ }^{2}$, S. Erten ${ }^{2}$ ${ }^{1}$ Rheumatology, Ankara Research and Education Hospital; ${ }^{2}$ Rheumatology, Yildirim Beyazit University, Ankara, Turkey

Background: Acute rheumatic fever (ARF) is a delayed, inflammatory sequela of pharyngitis secondary to Group A Streptococcus infection. ARF remains one of the most important causes of cardiovascular morbidity and mortality in developing countries. Although it is mainly known as a childhood disease, it is also encountered in adult clinics in developing countries.

Objectives: To investigate the clinical and laboratory characteristics of patients who were diagnosed with ARF in two rheumatology outpatient clinics from June 2015 to January 2017.

Methods: The data of 20 patients (12 female; median age 29.5 (21-40) years) were evaluated. The diagnosis of ARF was based on the 2015 Jones criteria. The data collected included patient age, gender, arthralgia, arthritis, erythema marginatum (EM), subcutaneous nodules (SN), ECG/ Doppler transthoracic echocardiography findings, and other rare findings. The erythrocyte sedimentation rate (ESR), antistreptolysin $\mathrm{O}$ (ASO) and CRP levels of the patients and the drugs initiated were also recorded. Anti-streptolysin O (ASO) test or throat culture were used for the evidence of preceding Streptococcus infection. Patients with post-streptococcal reactive arthritis were differentiated and excluded by clinically. Patients with positive rheumatoid factor or ACPA were also excluded. Joint fluid examination was done to exclude septic arthritis in patients with monoarthritis.

Results: All patients were referred to rheumatology for arthralgia or arthritis. Patients were taking some sort of nonsteroidal antiinflammatory (NSAI) drugs before the referral. The median follow-up time was 9 months $(0-18)$. Sixteen out of 20 patients had mono-, oligo- or poliarthritis (25\%,25\% and $30 \%$, respectively). Knees and ankles were the most common involved joints. The median duration of arthritis was 1 week (1-50 weeks). Six out 20 patients had subclinical carditis (30\%). Nine out of 20 patients had a history of ARF attack previously. Three patients had chronic rheumatic mitral valve thickening without any severe insufficiency. EM and SN were observed in $15 \%$ and $60 \%$ of patients, respectively. Chorea was diagnosed in one patient. NSAI drugs were given to all patients with maximum dosages. High dose salicylate therapy were not given to patients due to intolerance or side effects. Nine patients were given prednisolone therapy (5-20 $\mathrm{mg} / \mathrm{d}$ ). The median duration of prednisolone therapy was 2 weeks (0-6 weeks). Sulfasalazine was given to two patients for the prolonged arthritis. All patients received secondary prophylaxis with penicillin.

Conclusions: ARF should be considered in the differential diagnosis of arthritis in young adults in developing countries. Arthritis of ARF in adults seems to be resistant to classical NSAI drugs. Our data show that steroid therapy can be given safely instead of salicylates in carditis or arthritis.

\section{References:}

[1] GewitzMH,BaltimoreRS,TaniLY,etal. AmericanHeart Association Committee on Rheumatic Fever, Endocarditis and Kawasaki Disease of the Council on Cardiovascu- lar Disease in the Young. Revision of the Jones criteria for the diagnosis of the rheumatic fever in the era of Doppler echocardiography: a scientific statement of the American Heart Association. Circulation 2015; 131 : 1806-18.

[2] He VY, Condon JR, Ralph AP, et al. Long-Term Outcomes From Acute Rheumatic Fever and Rheumatic Heart Disease: A Data-Linkage and Survival Analysis Approach. Circulation. 2016 Jul 19;134(3):222-32.

Disclosure of Interest: None declared

DOI: 10.1136/annrheumdis-2017-eular.4944

\section{SAT0571 PERFORMING SEPTIC ARTHRITIS DIAGNOSIS IN SCENARIOS WHERE SYNOVIAL FLUID IS NOT AVAILABLE: MULTIVARIATE LOGISTIC REGRESSION ANALYSIS}

C.A. Guillen-Astete ${ }^{1}$, G.A. Gabilondo-Alvarez ${ }^{2} .{ }^{1}$ Rheumatology Department; ${ }^{2}$ Microbiology, Ramon y Cajal University Hospital, Madrid, Spain

Background: In order to establish the diagnosis of septic arthritis (SA) it is necessary to demonstrate the presence of bacteria into the synovial fluid. However, clinics should act according to its suspicious when the differential diagnosis included this possibility even when the microbiologic study of the synovial fluid in unavailable by any cause (small joints, lack of training or logistic deficiencies).

Objectives: The purpose of our study is to determine the relative weight of other clinical or analytical variables that should be useful in these scenarios.

Methods: A retrospective multivariate logistic regression analysis about the registries of monoarthritis assessed in our unit between 2013 and 2016. There were included only registries with cases of synovial joints with all parameters of interest available. The binary response variable was the microbiological demonstration of septic arthritis (culture/Gram stain of synovial fluid or tissue).
Explanatory variables were age (stratified in $<30,30-39,40-49,50-59,60-69$ and $>70$ years old), gender, temperature above of $37.9^{\circ} \mathrm{C}$, recount of neutrophils in peripheral blood sample, measure of procalcitonine (PCT), and measure of $C$ reactive protein (CRP). Synovial fluid study, although was available in almost all cases was deliberately omitted for purpose of this study. The logistic regression analysis used the forward model strategy.

Results: There were included 449 registries. One hundred an sisteen of them were SA. The fixed model showed a Chi-square=226.64 with 5 degrees of freedom and a $P<0.0001$. Explanatory variable gender was excluded from the forward model strategy due to its lack of impact on the binary response variable. The Odds Ratio for PCR, PCT $>1.3 \mathrm{ng} / \mathrm{dL}$, age, neutrofils recount and body temperature were $1.0175,8.1588,0.5135,1.0001$ and 3.4147 , respectively. The model showed a sensibility of $68.1 \%$, specificity of $94.8 \%$, PPV of $82.2 \%$ and NPV of $89.5 \%$. The following table shows the full results of the logistic regression analysis: Standard error, Wald index, $\mathrm{p}$ value and $\mathrm{R}$ coefficients.

\begin{tabular}{|llllll|}
\hline Variable & Coefficient & E. E. & $\begin{array}{l}\text { Chi2- } \\
\text { Wald }\end{array}$ & G.F. & p-value \\
\hline PCR & 0.0174 & 0.0024 & 52.0092 & 1 & $<0.0001$ \\
\hline PCT & 2.0991 & 0.3407 & 37.9640 & 1 & $<0.0001$ \\
\hline Age & -0.6665 & 0.1326 & 25.2763 & 1 & $<0.0001$ \\
\hline $\begin{array}{l}\text { Neutrophils } \\
\text { recount }\end{array}$ & 0.0001 & 0.0001 & 14.9630 & 1 & 0.0001 \\
\hline T>37.9 & 1.2281 & 0.3286 & 13.9674 & 1 & 0.0002 \\
\hline Constant & -3.7227 & - & - & - & N.A. \\
\hline
\end{tabular}

Conclusions: Previous studies have demonstrated the usefulness of PCT measure for the diagnosis of SA however all of them have been based on joints which synovial fluid is quite easy to obtain for further analysis. Present study allows laying the ground for creation of future diagnostic models based on five clinical variables that could be useful on scenarios where the assessment by joint puncture is not available.

Disclosure of Interest: None declared

DOI: 10.1136/annrheumdis-2017-eular.4240

\section{SAT0572 LEVELS OF SERUM PROCALCITONINE AS DIAGNOSIS DISCERNING TOOL BETWEEN GOUT AND SEPTIC ARTHRITIS}

C.A. Guillen-Astete, M. Terán-Tinedo. Rheumatology Department, Ramon y Cajal University Hospital, Madrid, Spain

Background: In a previous study we demonstrated the potential usefulness of the procalcitonine serum measure in order to diagnose septic arthritis. This utility is quite important on the differential diagnosis of knee arthritis in patients with known gout or other inflammatory joint disease which can express as a monoarthritis.

Objectives: Due to that, this study aims to determine sensibility, specificity, positive predictor value and negative predictor value of procalcinonine sereum measure (PCT) in patients with knee arthritis for the diagnosis of septic arthritis.

Methods: We reviewed the registries of patients who consulted due to knee acute monoarthritis between 2013 and 2015 in which a PCT was obtained. Registries were grouped according to the final diagnosis (gouty arthritis only (group I) and septic arthritis with or without gout (group II). Based on a previous study, PCT value of $1.47 \mathrm{ng} / \mathrm{mL}$ was considered the cut point. Validation tests were applied fixed to demographic and clinical specific scenarios.

Results: Registries of 121 patients with gout and 47 patients with septic arthritis (SA) were included. From the 47 patients with SA, 9 were previously diagnosed by gout. All diagnosed were based on guidelines and clinical recomendations for SA and gout (Coakley et al. Rheumatology, 2006 and Zhang et al. Ann Rheum Dis, 2006).

Using the cut point of $1.47 \mathrm{ng} / \mathrm{mL}$, test validation results were as follows: sensibility of $87.2 \%$, specificity of $92.5 \%$, PPV of $82.0 \%$ and NPV of $94.9 \%$. Excluding patients with less than 48 hours of onset, there were 44 registries of patients with SA and 83 with gout. In this scenario the results of the validation tests were as follows: Sensibility of $93.1 \%$, specificity of $96.3 \%$, PPV of $93.1 \%$ and NPV of $96.38 \%$. Excluding all patients with body temperature above $37.5^{\circ} \mathrm{C}$ there remained 11 with SA and 81 with gout. In this scenario the results of the validation tests were as follows: Sensibility of $72.7 \%$, specificity of $95.1 \%$, PPV of $66.6 \%$ and NPV of $96.2 \%$.

Conclusions: This is the first study aimed to validate previous observations about the usefulness of PCT determination in patients with acute knee monoarthritis. Altough the global results in the validation test are a bit inferior that our original observations in a shorter pilot study in terms of sensibility, the NPV remains over $95 \%$ in scenarios where the differential diagnosis could be harder to establish such as recent onset flares or cases without an associated febrile syndrome.

Disclosure of Interest: None declared

DOI: 10.1136/annrheumdis-2017-eular.4289 